\title{
Estudio de la propagación de Myrcianthes coquimbensis (Barnéoud) Landrum et Grifo por semillas y esquejes
}

\section{Propagation of Myrcianthes coquimbensis (Barnéoud) Landrum et Grifo by seeds and cuttings}

\author{
Gabriela Saldías* \& Juan Velozo
}

Universidad Central de Chile, Facultad de Arquitectura, Urbanismo y Paisaje, Escuela de Arquitectura del Paisaje. Santa Isabel 1186, Santiago, Chile.

*mgsaldiasp@ucentral.cl

\begin{abstract}
RESUMEN
Myrcianthes coquimbensis es una especie endémica de Chile en peligro de extinción, con una distribución restringida en la costa de la Región de Coquimbo. En la actualidad su hábitat está siendo fuertemente impactado por desarrollos inmobiliarios. La especie presenta valor ornamental; sin embargo, desde el punto de vista paisajístico es poco conocido. En esta investigación se estudió la propagación por semillas y vegetativa por esquejes, con fines de conservación ex situ. En ensayos de germinación se encontró que la cinética de este proceso varió significativamente según la época de siembra (invierno o verano). Así después de 90 días de siembra se observó un 51\% de germinación en verano, mientras en invierno sólo alcanzó el 29\%. También se observó un efecto inhibitorio del pericarpio sobre la germinación, disminuyendo un 50\% la germinación. La incubación de semillas en $\mathrm{GA}_{3}(24 \mathrm{~h})$ incrementó el porcentaje de germinación dependiendo de la dosis. Se realizaron ensayos de enraizamiento de esquejes con tratamientos de AIB en cama fría y cama caliente. En cama fría se observó una baja respuesta $(8,44 \%)$, y no mostró relación con los tratamientos de AIB. En contraste a lo anterior, en cama caliente el enraizamiento alcanzó un 33\% con aplicación de 3.000 ppm de AIB. El mejor resultado de enraizamiento se obtuvo con material proveniente de plantas mantenidas en condiciones de vivero.
\end{abstract}

Palabras clave: Myrtaceae, flora amenazada, endemismo chileno, conservación ex situ.

\begin{abstract}
Myrcianthes coquimbensis is an endangered species, endemic from Chile where it has a restricted distribution in the coast of the Coquimbo Region. Nowadays its habitat is being heavily impacted by real estate development. This species has ornamental value, but is almost unknow from gardeners and landscapers. This work aims at contributing to Myrcianthes coquimbensis ex situ conservation by development of its propagation by both sexual and vegetative way. Germination tests shown that the kinetics of this process varied significantly according to the sowing season (winter or summer). After 90 days, emergence was of $51 \%$ in summer, while in winter it only reached $29 \%$. The pericarp was found to have an inhibitory effect, decreasing germination by $50 \%$. Incubation of seeds in $\mathrm{GA}_{3}$ during $24 \mathrm{~h}$ increased the percentage of germination with a dose-dependent effect. Cutting-propagation assays were performed with AIB treatments in cold or warm bed. In cold bed, a poor response was obtained ( $8.44 \%$ of rooting), without influence of treatments. However, in hot bed, rooting reached $33 \%$ with application of $(3,000 \mathrm{ppm})$. Best results were obtained when stems came from plants maintained under nursery conditions.
\end{abstract}

KeYwords: Myrtaceae, endangered, Chilean endemic, ex situ conservation.

\section{INTRODUCCIÓN}

Myrcianthes coquimbensis (Barnéoud) Landrum et Grifo (Myrtaceae) es un arbusto endémico, clasificado en condición de en peligro de extinción (Benoit 1989, Squeo et al. 2001).
Tiene una distribución muy restringida en la costa rocosa de la Región de Coquimbo (Squeo et al. 2001, Hechenleitner et al. 2005, Riedemann et al. 2006). Hechenleitner et al. (2005) señalan a La Higuera (29 28'S) como la distribución más septentrional y el límite sur ha sido señalado para la localidad de Guanaqueros $\left(30^{\circ} 12^{\prime} \mathrm{S}\right.$; 
$\left.70^{\circ} 30^{\prime} \mathrm{O}\right)$, comprendiendo aproximadamente $66 \mathrm{~km}$ de norte a sur y no más de $2 \mathrm{~km}$ hacia el interior (Riedemann et al. 2006, Squeo et al. 2008). García-Guzmán et al. (2012) señalan $82,8 \mathrm{~km}$ de extensión a lo largo de la costa chilena. En su hábitat el período seco dura 6 a 10 meses y las precipitaciones promedio de La Serena son $78 \mathrm{~mm}$ (Dirección Meteorológica de Chile, dic 2013).

La especie crece en formaciones vegetales que también se encuentran en situaciones de vulnerabilidad. Según Gajardo (1994) pertenece a la Región Vegetacional del Matorral y del Bosque Esclerófilo, en la Sub Región del Matorral Estepario, en formaciones de Matorral Estepario Costero y de Matorral Estepario Interior. Se asocia con Bridgesia incisifolia, Centaurea chilensis, Fuchsia lycioides, Llagunoa glandulosa, Ophryosporus triangularis, Oxalis gigantea y Proustia ilicifolia (Hechenleitner et al. 2005). Luebert \& Pliscoff (2006) la incluyen en la formación vegetal del Matorral Desértico y el piso vegetacional del Matorral Desértico Mediterráneo Costero de Oxalis gigantea y Heliotropium stenophyllum. En la actualidad el hábitat del lucumillo se encuentra muy perturbado por proyectos inmobiliarios - urbanísticos; y por intervenciones antrópicas (Saldías \& Velozo 2012). Las zonas más afectadas por esta situación son El Arrayán y El Panul (GarcíaGuzmán et al. 2012). Los terrenos son muy cotizados por la cercanía a grandes centros poblados. Otro problema ecológico importante es la extracción de tierra de hojas que se acumula bajo el follaje de los arbustos, lo que causa un daño a las plantas desde dos perspectivas, por un lado afecta la germinación de las semillas y el establecimiento de las plántulas y por otra parte disminuye el aporte de nutrientes afectando la fertilidad en suelos de zonas áridas. Además, para extraer la tierra de hojas muchas veces se dañan los arbustos al cortarlos desde la base o bien cortando las ramas basales. Este problema fue detectado en Caleta Hornos y Punta Teatinos (García-Guzmán et al. 2012). A esto se suma un megaproyecto minero en estudio que afectará las poblaciones de Myrcianthes coquimbensis localizadas en los sitios de intervención en Caleta Chungungo.

Desde el punto de vista del paisajismo el lucumillo es poco conocido, existen antecedentes de sólo dos viveros comerciales que han logrado su propagación por semilla. La especie se caracteriza por la belleza de su follaje siempreverde y aromático. Florece entre mayo y septiembre, y el fruto es una baya globosa (Fig. 1a) de color rojo en la madurez (Hechenleitner et al. 2005, Riedemann et al. 2006). La producción de frutos es muy variable según el año (Saldías \& Velozo 2012). Las semillas de Myrcianthes coquimbensis son recalcitrantes lo que corresponde a una característica de la familia de las Mirtáceas, por tanto una vez cosechadas se deben sembrar con prontitud. En las Mirtáceas es habitual la aplicación de tratamientos pregerminativos para eliminar condiciones de latencia, el procedimiento más utilizado es la maceración del fruto, consistente en la eliminación de las partes envolventes y remojo en agua, para eliminar posibles componentes causantes de latencia (Ramírez et al. 1980, Latsague et al. 2010). También se ha planteado el uso de giberelina como estimulante de la germinación en diversas especies (Araya et al. 2000, Atencio et al. 2003, Latsague et al. 2010). En esta investigación se realizaron ensayos de propagación con semillas y con esquejes, con el objetivo de determinar condiciones para su propagación. La finalidad de la investigación es aportar a la conservación $e x$ situ y fomentar el uso de la especie en obras de paisajismo, especialmente en jardines costeros.
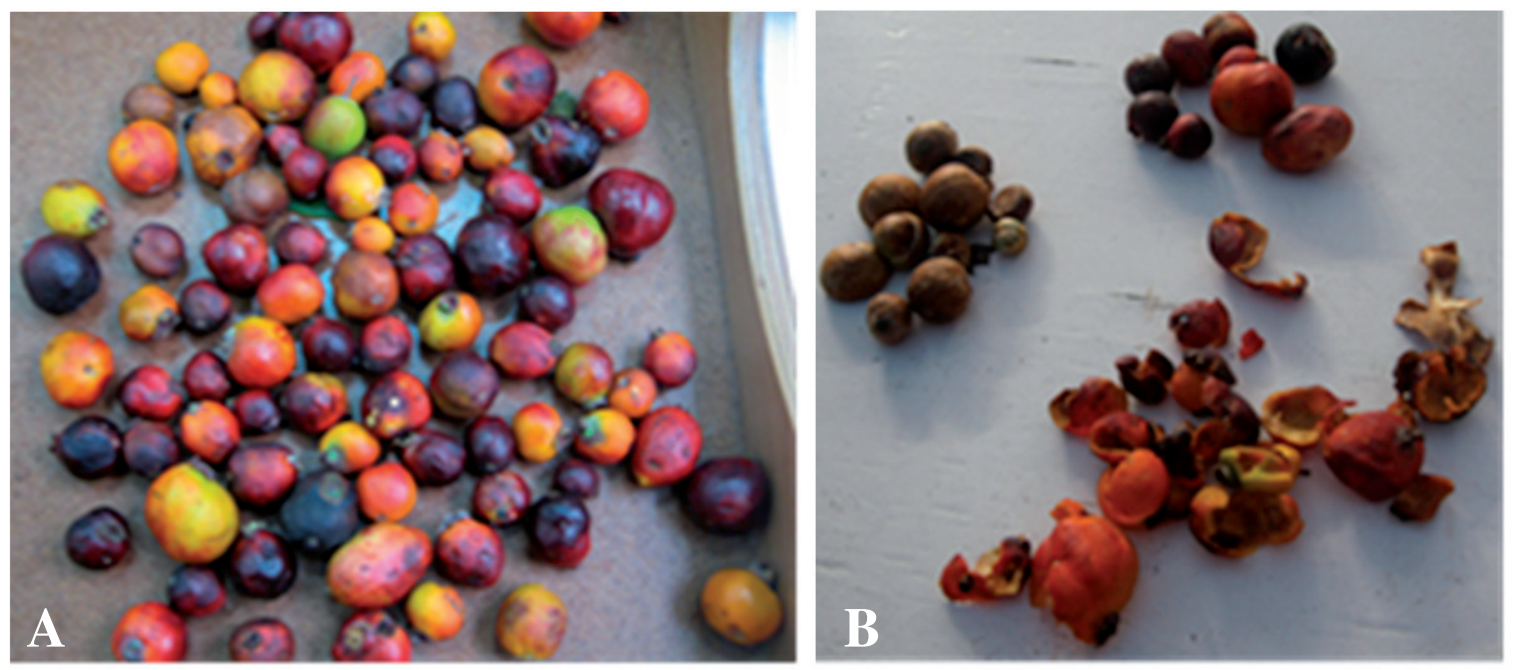

Figura 1. A. Variabilidad en tamaño y color de frutos. B. Retiro del pericarpio de frutos.

Figure 1. A. Variability in fruit size and color. B. Removal of the pericarp of fruits. 


\section{MATERIALES Y MÉTODOS}

\section{ENSAYOS DE GERMINACIÓN}

Durante el invierno y fines de primavera del año 2012 se recolectaron frutos en poblaciones silvestres de dos localidades, Punta Teatinos (Fig. 2) y Totoralillo. Los frutos se tomaron directamente de las plantas (10 individuos en cada localidad) en estado de madurez evidente y fácilmente desprendibles. En cada época de cosecha de frutos se realizaron siembras (agosto 2012 y enero 2013), con muestras triplicadas de 100 semillas. Para obtener las semillas, se eliminó la cubierta carnosa de los frutos (Fig. 1b) y se sometieron a maceración en agua (24 h). La siembra se realizó en bolsas de 10x15 (1,5 L) conteniendo una mezcla compuesta por: 2 partes de compost, 1 parte de fibra de coco, 1 parte de perlita. Se sembró una semilla por bolsa. Las pruebas fueron llevadas a cabo en un invernadero de policarbonato con ventilación cenital. Para determinar el efecto del pericarpio sobre la germinación, se realizó una siembra de frutos sin maceración (enero 2013). Los resultados fueron evaluados con la prueba de estadística $\mathrm{X}^{2}$

También se ensayaron tratamientos pregerminativos con ácido giberélico $\left(\mathrm{GA}_{3}\right)$. Para ello se embebieron semillas en solución de ácido giberélico $\left(\mathrm{GA}_{3}\right)$ durante $24 \mathrm{~h}$ en las siguientes concentraciones 0- 5- 10 - 15 y 20 g/L. En cada ensayo se midió la germinación en el tiempo. Se aplicó un diseño experimental aleatorio donde la unidad muestral fueron 20 semillas con tres repeticiones.

La siembra se realizó en tubetes de plástico duro, aérotubo, de $180 \mathrm{ml}$ de capacidad con grietas laterales que permiten una buena aireación del sustrato y facilidad de retirar las plantas una vez enraizadas con una mezcla igual a la anterior.

Las observaciones se realizaron durante 90 días y se compararon los resultados de germinación final con la prueba de $\mathrm{X}^{2}$

\section{Preparación de esqueJes}

Para obtener esquejes se cortaron ramas provenientes de plantas silvestres y de plantas cultivadas y mantenidas en vivero. Las muestras silvestres se recolectaron en

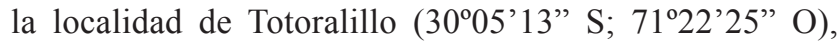
durante el mes de julio de 2011. Las ramas se colocaron en turba húmeda para su transporte en una caja fría y los esquejes fueron preparados dentro de las $48 \mathrm{~h}$ de la recolección. Los esquejes consistieron en secciones binodales, tanto de posición apical como subapicales, y de $5 \mathrm{~cm}$ de longitud aproximada (Tapia 1980, Soto 2004). En todos los casos se eliminaron las hojas inferiores y en la base del esqueje se realizó una lesión longitudinal en la peridermis de aproximadamente $1 \mathrm{~cm}$ para aumentar la superficie de absorción de la hormona. Como medida preventiva los esquejes fueron sumergidos en una solución fungicida (extractos de cítricos). Los ensayos se realizaron en cama fría y en cama caliente $\left(20^{\circ} \mathrm{C}\right)$ bajo túnel plástico con sistema de control de humedad a $80 \%$, mediante la activación de nebulizadores. Para estimular la formación de raíces se utilizó ácido indol butírico (AIB) en solución alcohólica a partir de una mezcla comercial (IBA Root Yates $\left.{ }^{\circledR}\right)$. Los tratamientos y concentraciones de AIB fueron T0: testigo, T1: 1.500, T2: 3.000, T3: 5.000 y T4: 7.500 ppm. El sustrato de enraizamiento estuvo compuesto por

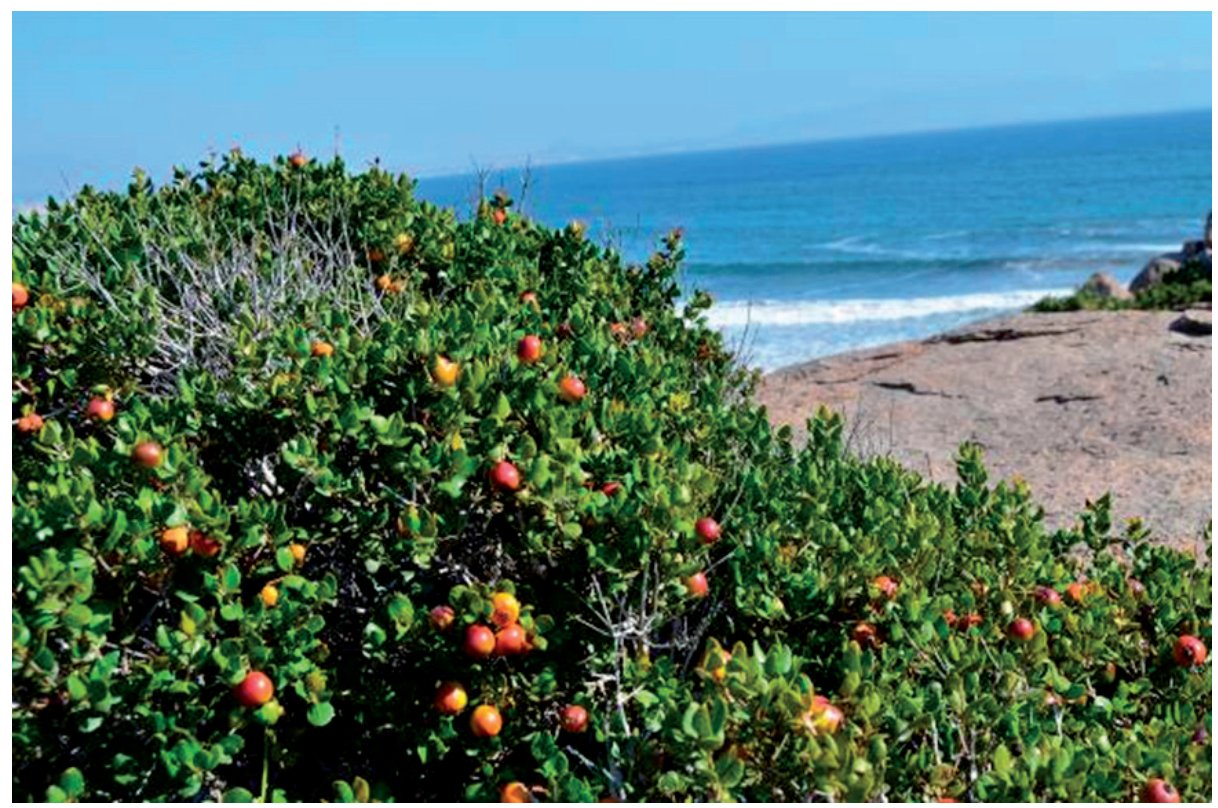

Figura 2. Lucumillos en Punta Teatinos.

Figure 2. Lucumillos in Punta Teatinos. 
una mezcla en partes iguales de arena gruesa tipo lampa, perlita y vermiculita. Las observaciones se realizaron desde los 45 a 140 días, momento en que los esquejes se retiraron del sustrato y se midieron las variables: presencia de raíz, número y largo de raíces.

La unidad muestral fueron 30 esquejes con 3 repeticiones en un diseño experimental completamente aleatorio. Las diferencias entre tratamientos fueron evaluadas con la prueba de $\mathrm{X}^{2}$. Para procesar los datos se utilizó el programa Minitab.

\section{RESULTADOS}

Al momento de la recolección de frutos, especialmente a fines de primavera, se pudo observar que algunos ya habían iniciado la germinación en la planta madre y presentaban la radícula expuesta. Esta característica aumentó una vez que permanecieron semillas en el laboratorio en espera de ser sembradas. Los frutos presentan distintos tamaños y contienen entre 1 a 2 semillas en una gran mayoría, excepcionalmente 3 ó 4 semillas pequeñas.

\section{GERMINACIÓN SEGÚN ÉPOCA DE SIEMBRA}

En la siembra invernal el inicio de la germinación se produjo a los 58 días a diferencia de la siembra en verano que se inició a los 15 días. El porcentaje de germinación fue de $29 \%$ a los 90 días de observación para la siembra invernal y de $51 \%$ para la siembra de verano (Fig. 3). Se observó mayor rapidez en la germinación en los primeros 3 meses en la siembra de verano, lo que se relaciona con mayor temperatura. Sin embargo, el porcentaje máximo de semillas germinadas fue de $82 \%$ para la muestra de invierno y de $53 \%$ para la muestra de verano.

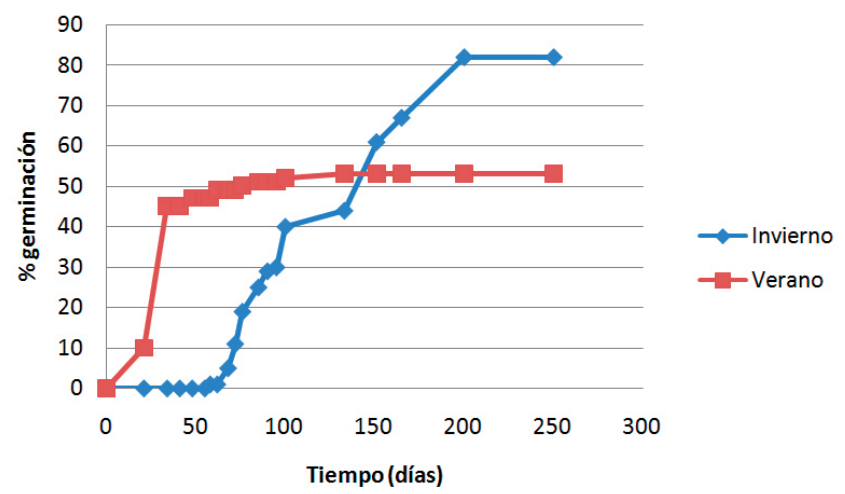

FIGURA 3. Efecto de la temporada de colecta y siembra de semillas de Myrcianthes coquimbensis en la respuesta de germinación.

FIGURE 3. Effect of collection and sowing season on seed germination of Myrcianthes coquimbensis.
MACERACIÓN DE FRUTOS

Las semillas desprovistas de pericarpio y maceradas germinaron antes y el porcentaje de germinación fue mayor respecto a la siembra con pericarpio. A los 30 días de la siembra germinó el $45 \%$ de las semillas en comparación con $5 \%$ de los frutos enteros. A los 100 días la germinación de las semillas maceradas fue el doble respecto a los frutos, $\chi^{2}$ $=14,2(\mathrm{p}=0,001)$ (Fig. 4).

\section{CRECimiento de PLANTAS}

A 200 días (cerca de 7 meses) se midió la altura alcanzada por las plantas.

La altura máxima alcanzada fue de $40 \mathrm{~cm}$ con un total de 34 hojas por tallo. La mayor frecuencia de altura estuvo comprendida entre 7 y $16 \mathrm{~cm}$ (Fig. 5, 6).

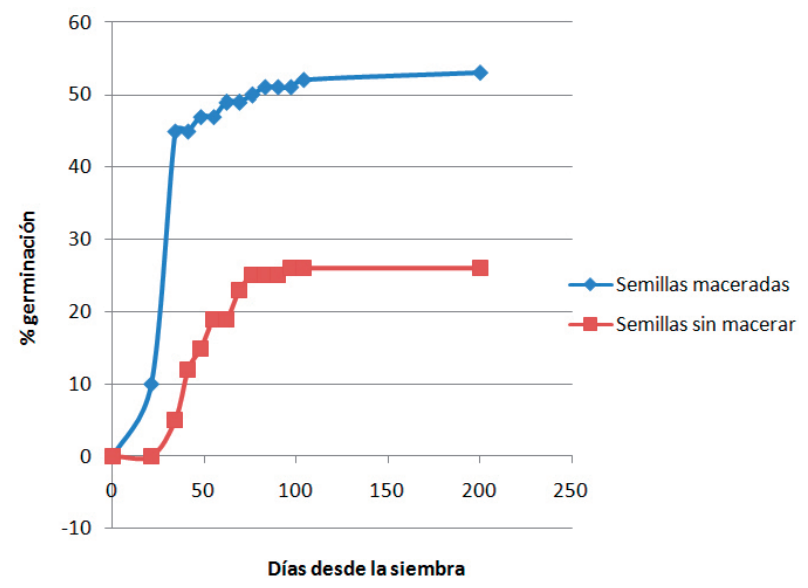

FIGURA 4. Efecto del pericarpio en la germinación de Myrcianthes coquimbensis.

FIGURE 4. Effect of pericarp in Myrcianthes coquimbensis germination.

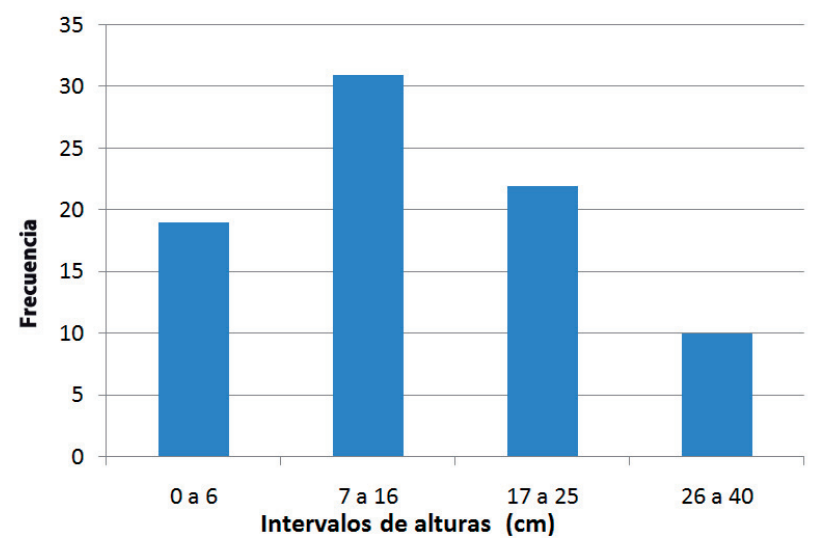

Figura 5. Distribución de alturas a los siete meses de la siembra. Promedio: 14,1 cm; DS 9,99; r (2-40).

FIgURE 5. Height distribution at seven months after sowing. Average: $14.1 \mathrm{~cm}$, SD 9.99, r (2-40). 


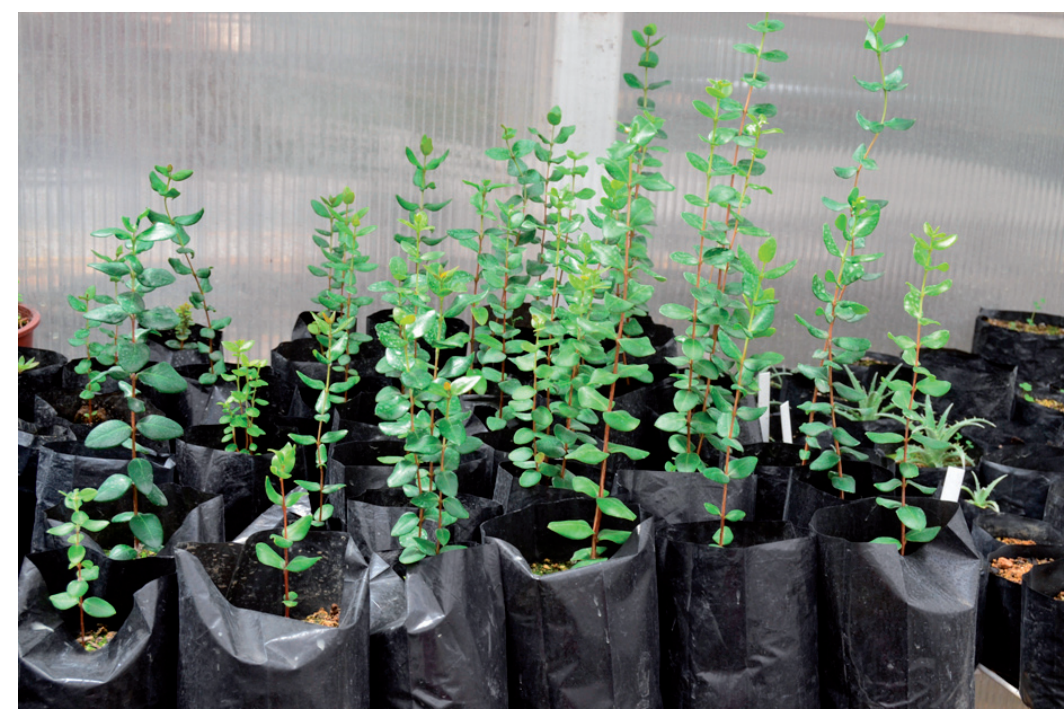

Figura 6. Plantas de lucumillos de 7 meses.

FIgURE 6. Lucumillos plants 7 months.

TRATAMIENTO PRESIEMBRA CON ÁCIDO GIBERÉLICO

Con fines de homogeneizar la germinación de las semillas fueron sometidas a tratamientos pregerminativos con solución de ácido giberélico por $24 \mathrm{~h}$. El Tratamiento de $15 \mathrm{~g} / \mathrm{L}$ de ácido giberélico incrementó significativamente el porcentaje de germinación $\chi^{2}=10,182(\mathrm{p}=0,037)$, alcanzando un 68,25\% (Fig. 7).

ENRAIZAMIENTO DE ESQUEJES

El material recolectado en terreno mostró un rápido decaimiento y oxidación por lo que los ensayos se realizaron con plantas viverizadas y mantenidas en esta condición. El enraizamiento de esquejes mantenidos en condiciones de

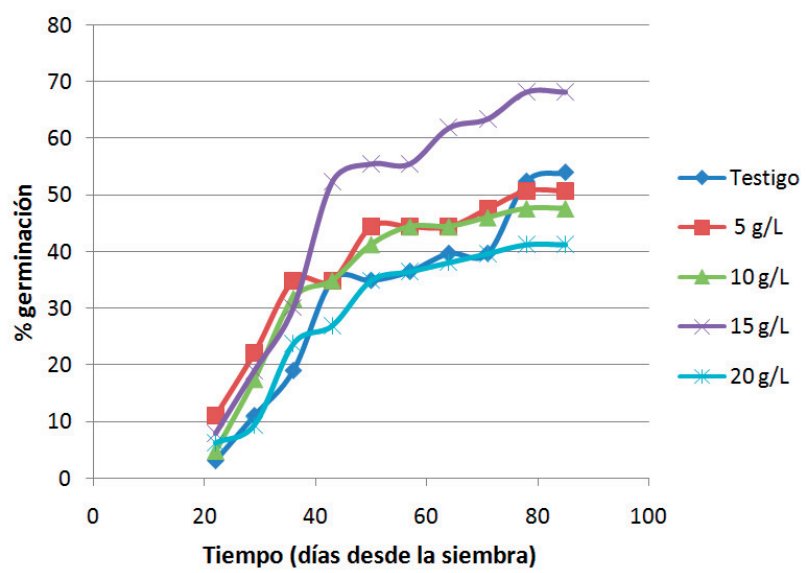

FiguRA 7. Efecto del ácido giberélico en la germinación de Myrcianthes coquimbensis.

FIGURE 7. Effect of gibberellic acid on the germination of Myrcianthes coquimbensis. cama fría presentó un bajo porcentaje de enraizamiento (promedio de 8,44\%) y no se observó diferencia entre tratamientos hormonales. La respuesta de enraizamiento mejoró en condiciones de cama caliente $\left(20^{\circ} \mathrm{C}\right)$ y control de humedad ambiental en $80 \%$. El máximo de enraizamiento se logró con la aplicación de 3.000 ppm de AIB, alcanzando un 33,33\% (Fig. 8, 9).

Los tratamientos de AIB, T1 (1.500), T2 (3.000) y T4 (7.500) difirieron significativamente comparados con el grupo control (T0) y respecto a la condición de cama fría, $\chi^{2}$ $=12,436(\mathrm{p}=0,014)$.

Respecto al número y largo de las raíces generadas en los distintos tratamientos no se observaron diferencias sustantivas (Tabla I).

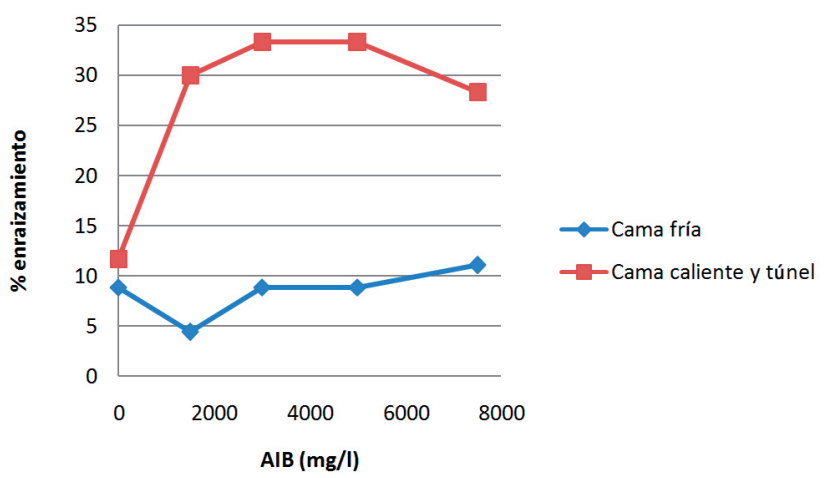

Figura 8. Efecto de las dosis de AIB y condición de incubación en el enraizamiento de Myrcianthes coquimbensis.

FIGURE 8. Effect of AIB and incubation condition in Myrcianthes coquimbensis rooting. 
TABLA I. Efecto de los tratamientos hormonales en el número y largo de raíces en esquejes mantenidos en túnel y cama caliente.

TABLE I. Effect of hormone treatments on the number and length of roots in cuttings held in tunnel and warm bed.

\begin{tabular}{|c|c|c|}
\hline $\begin{array}{l}\text { APLICACIÓN HORMONAL } \\
\text { AIB ppm }\end{array}$ & $\mathrm{N}^{\circ}$ RAícES PROMEDIO Y RANGO & $\begin{array}{l}\text { LARGO DE RAÍCES PROMEDIO Y RANGO } \\
\qquad(\mathrm{cm})\end{array}$ \\
\hline Testigo & 1,3 r (1-3) & 3,3 r $(2-6)$ \\
\hline 1.500 & $1,3 \mathrm{r}(1-3)$ & $3,3 \mathrm{r}(1,5-7)$ \\
\hline 3.000 & $1,4 \mathrm{r}(1-2)$ & $3,1 \mathrm{r}(1-6)$ \\
\hline 5.000 & $1,1 \mathrm{r}(1-2)$ & 3,0 r $(2-5)$ \\
\hline 7.500 & 1,7 r (1-3) & 3,5 r (3-4) \\
\hline
\end{tabular}

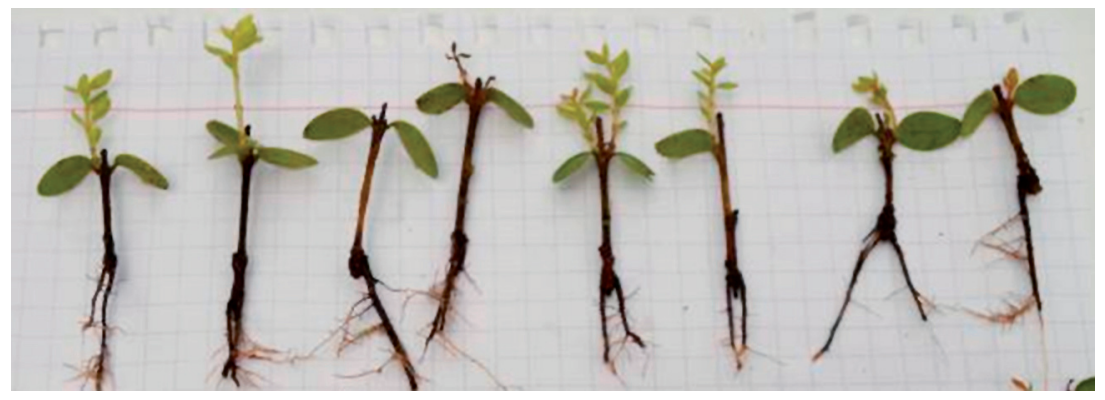

FIGURA 9. Enraizamiento de esquejes de lucumillo con aplicación de 3.000 ppm AIB.

FIGURE 9. Lucumillo rooting cuttings with 3,000 ppm application.

\section{DISCUSIÓN}

La germinación fue rápida en la siembra de verano lo que se relacionaría con mayor temperatura, estabilizándose desde los 90 días, mientras en invierno, la germinación fue lenta pero con una progresión lineal hasta el final del periodo ( $250 \mathrm{~d})$. Esta respuesta es muy interesante ya que el periodo de fructificación en la especie es muy largo, en un hábitat donde las precipitaciones son ocasionales. El porcentaje máximo de semillas germinadas difirió para cada muestra de semillas dependiendo de la fecha de recolección, la muestra de invierno fue recolectada en junio y sembrada inmediatamente, en cambio la muestra de verano fue colectada en noviembre y sembrada dos meses después, en enero (lo que pudo haber generado pérdidas de viabilidad de semillas). Es interesante destacar que en la recolección de verano un porcentaje importante de semillas había iniciado la germinación en la planta, lo que se asocia principalmente con la mayor temperatura de la época. Es decir que esta semilla sería de germinación rápida y sincrónica al final del periodo de fructificación.

Se encontró un marcado efecto inhibitorio del pericarpio en la germinación, lo cual podría estar relacionado con compuestos fenólicos existente en estos tejidos, que le dan un color rojizo y luego parduzco al fruto. El ácido giberélico tiene efecto positivo en la germinación de esta especie aumentando la capacidad germinativa en un $20 \%$, esto ha sido descrito para otras especies con semillas recalcitrantes (Araya et al. 2000, Atencio et al. 2003, Latsague et al. 2010). Respecto a las plantas generadas, las alturas a los 7 meses presentan una distribución normal, tienen principalmente un crecimiento monopodial, excepcionalmente se producen plantas con 2 o 3 tallos desde la base. No se observó caída de almácigos.

Los esquejes preparados a partir de material recolectado en terreno no lograron enraizar, lo que se puede explicar por el alto grado de estrés hídrico en que se encontraban las plantas al momento de la recolección de material. Las mediciones realizadas en material de vivero mostraron una clara diferencia en la respuesta al enraizamiento según las condiciones ambientales. Las condiciones de alta humedad ambiente y temperatura en el sustrato (túnel de niebla y cama caliente) favorecen el enraizamiento, sin embargo los efectos sobre el largo y número de raíces no difieren significativamente entre los tratamientos. También se evidenció el efecto positivo del ácido indol butírico en el enraizamiento. El repique de los esquejes desde la cama de enraizamiento a un nuevo contenedor detiene el crecimiento de los esquejes, causando un alto porcentaje de pérdidas, 
lo que hace necesario asegurar el traslado evitando que las raíces queden al aire. Coincide con los resultados obtenidos con Amomyrtus luma, Amomyrtus meli y Luma apiculata que no obstante de producirse la rizogénesis el porcentaje de sobrevivencia de esquejes fue baja (Soto 2004).

\section{CONCLUSIONES}

Sobre la base de los resultados de esta investigación es posible establecer que el uso de la semilla es una forma segura de obtener plantas con un buen desarrollo radicular. La maceración de los frutos y tratamiento pregerminativo con ácido giberélico son procedimientos efectivos para anticipar y uniformar germinación. Sería beneficioso determinar áreas de producción de semillas (APS), las que se debieran seleccionar por las respuestas germinativas y vigor de plantas producidas, entre las principales poblaciones silvestres de la especie.

Respecto a la propagación vegetativa, si bien la respuesta al enraizamiento es exigente en cuanto a requerimientos ambientales, es posible de lograr y permite ser aplicada para la conservación de genotipos destacados.

\section{AGRADECIMIENTOS}

Se agradece la colaboración de la Sra. Paz Acuña por su activa participación y valioso aporte de ideas en la realización de los ensayos experimentales. Esta investigación fue financiada por el Fondo de Investigación de la Universidad Central de Chile, año 2011-2012 y 2013 -2014.

\section{BIBLIOGRAFÍA}

Araya, E., L. Gómez, N. Hidalgo \& R. Valverde. 2000. Efecto de la luz y del ácido giberélico sobre la germinación in vitro de Jaul (Alnus acuminata). Agronomía Costarricense 24(1): 75-80.

Atencio, L., R. Colmenares, M. Ramírez \& D. Marcano. 2003. Tratamientos pregerminativos en acacia San Francisco (Peltophorum pterocarpum) Fabaceae. Universidad del Zulia, Venezuela. Revista Facultad de Agronomía 20: 63-71. http://www.scielo.org.ve/scielo.php?pid=S037878182003000100007\&script=sci_arttext

Benoit, I. (ed.). 1989. Libro Rojo de la Flora terrestre de Chile. Corporación Nacional Forestal. Ministerio de Agricultura. Santiago, Chile. 157 pp.

Dirección Meteorológica de Chile. Dirección de aeronáutica civil. Consulta diciembre 2013. http://www.meteochile. gob.cl/inf_precipitacion.php.

Gajardo, R. 1994. La vegetación natural de Chile. Clasificación y distribución geográfica. Santiago, Chile. Editorial Universitaria. $165 \mathrm{pp}$.
García-Guzmán, P., A.P. Loayza, D.E. Carvajal \& F.A. SQueo. 2012. The ecology, distribution and conservation status of Myrcianthes coquimbensis: a globally endangered endemic shrub of the Chilean Coastal Desert. Plant Ecology \& Diversity 5(2): 197-204. http://www.ieb-chile. cl/uploads/publicaciones/-1_Garcia-Guzman_etal_2012_ Plant_Ecol_Diver.pdf

Hechenleitner, P., M.F. Gardner, P.I. Thomas, C. Echeverría, B. Escobar, P. Brownless \& C. Martínez. 2005. Plantas amenazadas del Centro-Sur de Chile. Distribución, Conservación y Propagación. Primera Edición. Universidad Austral de Chile y Real Jardín Botánico de Edimburgo. 188 pp. http://issuu.com/chilebosque/docs/ hechenleitner.et.al.2005.plantas_amenazadas_chile

LANDRUM, L.R \& F.T. GRIFFo. 1988. The myrtle family (Myrtaceae) in Chile. Proceedings of the California Academy of Science 45: 277-317.

Latsague, M., P. Sáez \& L. Coronado. 2010. Tratamientos pregerminativos para Myrceugenia exsucca (Myrtaceae). Bosque (Chile) 31(3): 243-246. http://www.scielo.cl/scielo. php?script=sci_arttext\&pid=S0717-92002010000300008

Luebert, F. \& P. Pliscoff. 2006. Sinopsis bioclimática y vegetacional de Chile. Santiago, Chile. Editorial Universitaria. 316 pp.

Ramírez, C., M. Romero \& O. Henríquez. 1980. Estudio de germinación en semillas de Mirtáceas chilenas. Bosque (Chile): 3(2) 106-114. http://mingaonline.uach.cl/scielo. php?pid=S0717-92001980000100006\&script=sci_arttext

Riedemann, P., G. Aldunate \& S. Teillier. 2006. Flora nativa de valor ornamental. Zona Norte. Edición 1, Chile. 405 pp.

Saldías, G. \& J. Velozo. 2012. Distribución geográfica y estado de las poblaciones de Myrcianthes coquimbensis (Myrtaceae), especie endémica de Chile, en peligro de extinción. Chloris Chilensis Año 15, N². http://www. chlorischile.cl/Myrcianthes-saldias/saldias-lucumillo\%20 rev\%20st-rev\%20gs-22-12.htm

Squeo, F., G. Arancio \& J. Gutiérrez (eds.). 2001. Libro Rojo de la Flora Nativa y de los Sitios Prioritarios para su Conservación: Región de Coquimbo. Ed. Universidad de La Serena. Chile. 372 pp.

Squeo, F.A., Y. Tracol, D. López, M. León \& J.R. Gutiérrez. 2008. Vegetación nativa y variación temporal de su productividad en la Provincia de Elqui. En: P.J. Cepeda (ed.), Los Sistemas Naturales de la Cuenca del Río Elqui (Región de Coquimbo, Chile): Ediciones Universidad de La Serena, La Serena, Chile. p. 168-191. http://www.parc. $\mathrm{ca} / \mathrm{mcri} /$ pdfs/books/cepeda/4.pdf

Soто, P. 2004. Reproducción vegetativa por estacas en Amomyrtus luma, Amomyrtus meli y Luma apiculata mediante el uso de plantas madres jóvenes y adultas. Tesis para optar al título de Ingeniero Forestal. Universidad Austral de Chile. Valdivia, Chile. 64 pp. http://www.arboretum.cl/ Descargas/fifs718r.pdf

TAPIA, J. 1980. Efectos del medio de propagación en el enraizamiento de claveles (Dianthus caryophillus) cv. Sir Arthur Sim e influencia del desarrollo radicular al momento del transplante sobre el crecimiento de la planta. En: Recopilación. Universidad Católica de Valparaíso, Quillota, Chile. p 1-9.

Recibido: 21.08 .13

Aceptado: 31.12 .13 